\title{
Decoding the Patterns of Self and Nonself by the Innate Immune System
}

\author{
Ruslan Medzhitov* and Charles A. Janeway Jr.
}

\begin{abstract}
The innate immune system evolved several strategies of self/nonself discrimination that are based on the recognition of molecular patterns demarcating infectious nonself, as well as normal and abnormal self. These patterns are deciphered by receptors that either induce or inhibit an immune response, depending on the meaning of these signals.
\end{abstract}

The innate immune system of vertebrate animals uses three strategies of immune recognition that can be described in terms of recognition of "microbial nonself," recognition of "missing self," and recognition of "induced or altered self." The basis of microbial nonself recognition lies in the ability of the host to recognize conserved products of microbial metabolism that are unique to microorganisms and are not produced by the host. This strategy allows the innate immune system to discriminate between "infectious nonself" and "noninfectious self."

The second strategy, recognition of "missing self," relies on the detection of "markers of normal self." Such recognition is coupled with various inhibitory pathways that block initiation of immune responses against self. Markers of normal self are dedicated gene products and products of metabolic pathways that are unique to the host and absent from microorganisms.

The third strategy, recognition of induced self is based on the detection of markers of abnormal self that are induced upon infection (in particular, viral infection) and cellular transformation. Markers of abnormal self tag the affected cells for elimination by the immune system.

\section{Recognition of Microbial Nonself}

Recognition of microbial nonself, which evolved early in metazoan evolution, plays a crucial role in host defense (1). This recognition strategy is based on the detection of conserved molecular patterns that are essential products of microbial physiology. These invariant structures are referred to as pathogen-associated molecular patterns (PAMPs), although they are not unique to pathogens and are produced by all microorganisms, pathogenic or not (see below). PAMPs are unique to microbes (and are not produced by the host), and invariant among microorganisms of a given class $(1,2)$. The best known

Howard Hughes Medical Institute and Section of Immunobiology, Yale University School of Medicine, New Haven, CT 06520, USA.

*To whom correspondence should be addressed. Email: ruslan.medzhitov@yale.edu examples of PAMPs include lipopolysaccharide (LPS) of gram-negative bacteria and peptidoglycan of gram-positive bacteria. These and other PAMPs are recognized by receptors of the innate immune system called pattern recognition receptors (PRRs). Because PAMPs are produced only by microorganisms, they are perceived by the innate immune system as molecular signatures of infection, and their recognition by PRRs leads to the induction of an immune response $(1,2)$. Recognition of PAMPs thus allows the immune system to distinguish self from microbial nonself.

Several structurally and functionally distinct classes of PRRs evolved to recognize PAMPs and to induce various host defense pathways. Secreted PRRs bind to microbial cells and flag them for destruction either by the complement system or by phagocytosis. Toll-like receptors (TLRs), which comprise the class of PRRs expressed on the cell surface, activate signaling pathways that induce antimicrobial effector responses and inflammation upon recognition of PAMPs (3). In addition, activation of TLRs expressed on specialized antigen-presenting cells (APCs), such as dendritic cells, plays a critical role in the initiation of the adaptive immune responses (3). Triggering TLRs on these cells induces the expression of costimulatory molecules on the cell surface, which is necessary for the activation of naïve $T$ cells specific for antigenic peptides expressed on the same APC in complex with major histocompatibility complex (MHC) molecules. Because the costimulators are induced by PAMPs, their expression on APCs flags the antigenic peptides presented by the same APC as being of microbial origin and activates antigen-specific $T$ cells. Self-peptides expressed and presented by APCs are not recognized as nonself, because $T$ cells specific for these peptides are eliminated during negative selection in the thymus. Thus negative selection and microbial induction of costimulatory molecules together ensure that the adaptive immune response is generated against infecting pathogens but not against self-antigens (Fig. 1).
Evolution of Recognition in Host-Pathogen Interactions

Host-pathogen interactions involve multiple molecular recognition events that are required for productive infection by the pathogen or for resistance against infection by the host. These recognition events involve one or more components encoded by the pathogen (let's call them P) and one or more components encoded by the host (let's call them H). It is important to distinguish between two alternative evolutionary scenarios that led to these interactions. In one scenario, $\mathrm{H}$ evolved to recognize $\mathrm{P}$, while $\mathrm{P}$ evolved to perform functions unrelated to hostpathogen interactions. In this scenario, $\mathrm{H}$ is typically a PRR (for example, TLR4), and P is a PAMP (for example, LPS). In animals with adaptive immune systems, $\mathrm{H}$ can also be an antigen receptor, in which case $\mathrm{P}$ can be any microbial antigen. In the second scenario, $\mathrm{P}$ evolved to recognize $\mathrm{H}$, while $\mathrm{H}$ evolved to perform functions unrelated to this interaction. In this scenario, $\mathrm{P}$ interacts with $\mathrm{H}$ because this interaction is necessary for the productive infection of the host by the pathogen. In this case, $\mathrm{P}$ is usually referred to as a virulence factor (especially in the case of bacterial pathogens). Examples of interactions of this type are the recognition of human $\mathrm{E}$ cadherin by internalin A (a virulence factor of Listeria monocytogenes), and the recognition of CD4 by HIV gp120. In a distinct class of host-pathogen interactions in which the host and the microorganism both benefit from an interaction, $\mathrm{P}$ and $\mathrm{H}$ may have coevolved to interact with each other.

It is important that PRRs do not distinguish between microorganisms that colonize the host (pathogenic or commensal) and microorganisms that evolved to occupy habitats other than the host, because all of them produce PAMPs (3). However, only pathogens evolved the means to gain access to the compartments within the host where the host's PRRs can detect them and can induce immune responses.

\section{Molecular Passwords of Self-Identity}

The concept of missing self was proposed to explain why natural killer (NK) cells preferentially kill target cells that express few or no MHC class I proteins on the cell surface (4). On the basis of these studies, it has been suggested that NK cells recognize the lack of self MHC-I as a signal for target cell lysis (4). As MHC-I is constitutively expressed on all nucleated cells and is often down-regulat- 


\section{REFLECTIONS ON SELF: IMMUNITY AND BEYOND}

ed as a result of viral infection or cellular transformation, recognition by NK cells of a missing self-ligand allows them to selectively eliminate infected and transformed cells and to spare normal, healthy cells. The mechanism of missing-self recognition was worked out in studies that demonstrated that MHC-I is recognized by various inhibitory receptors, that upon binding to their ligands block the lytic activity of NK cells $(5,6)$. Inhibitory receptors expressed on $\mathrm{NK}$ and other cell types are characterized by a sequence motif called ITIM (immunoreceptor tyrosine inhibitory motif). When the tyrosine in the ITIM is phosphorylated, it recruits protein tyrosine phosphatases SHP-1 and SHP-2, which in turn inhibit cytotoxic activity by dephosphorylating tyrosine residues critical for activating NK cells $(5-8)$.

Recognition of missing self is not unique to NK cell function and is in fact widely used by the innate immune system. Another wellknown example of the use of this strategy is the regulation of the alternative complement pathway (9). A key component of the complement, a serum protein called $\mathrm{C} 3$, can covalently and indiscriminately attach itself to the surfaces of autologous and microbial cells. This attachment occurs as a result of a spontaneous hydrolysis of a highly labile thioester bond in C3. However, the formation of an active protease complex of the alternative pathway, the $\mathrm{C} 3$ convertase, occurs only on the surfaces of microbial cells and not on autologous cells (9). This is because all self cells ubiquitously express several gene products, including CD46 and CD55, which inhibit formation of the active $\mathrm{C} 3$ convertase (9). As cells of nonself origin, including pathogens, lack these host gene products, activation of the complement cascade can proceed uninhibited on their surfaces and results in lysis or phagocytosis of microbial cells (9). Because these inhibitors of complement are encoded exclusively by the genome of the host, they function as markers of self, or molecular passwords known only to the host.

Another example of a molecular signal that functions as a marker of normal self is the carbohydrate structures that decorate glycoproteins and glycolipids on the cell surface. In vertebrate animals, these usually terminate with sialic acids, which can be recognized by a variety of receptors involved in intercellular communication $(10,11)$. One group of receptors that can bind to sialated glycoproteins and glycolipids, but not to their desialated counterparts, comprise a family of proteins known as Siglecs (10). Similar to other inhibitory receptors, Siglecs are transmembrane proteins that contain ITIM motifs in their cytoplasmic regions. Although the function of most Siglecs is unknown, their structure and specificity for sialic acids suggest that they negatively regulate the function of leu- kocytes by monitoring the expression of sialic acids on normal self-tissues and antigens (10). Lack of sialic acid expression on most microorganisms, and in some cases, on virally infected and transformed cells may be recognized as a missing-self signal. Siglecs expressed on macrophages, dendritic cells, and neutrophils may direct these cells to phagocytose microbial nonself (which lacks biosynthetic pathways for sialic acids) and abnormal self (which lost sialic acid expression because of infection, transformation, or senescence). Another Siglec called CD22 is expressed on B cells and presumably helps to prevent unwanted antibody responses against self-antigens by blocking B cell receptor signaling (12). Sialic acids also regulate the activation of the alternative complement cascade by binding to a serum protein called factor $\mathrm{H}$, which upon binding to sialic acids on autologous normal cells inhibits formation of the C3 convertase (9).

Another, more recently discovered marker of self that regulates macrophage phagocytosis is CD47 (13). CD47 is constitutively expressed on many cell types, including erythrocytes, and was found to be a ligand for $\operatorname{SIRP} \alpha$, an ITIM-containing inhibitory receptor expressed on macrophages (13). Ligation of SIRP $\alpha$ by CD47 prevents phagocytosis of normal cells. Senescent erythrocytes, however, down-regulate CD47 expression and are effectively phagocytosed and removed from the circulation by splenic macrophages (13).

The above examples illustrate the common features of the missing-self strategy of immune recognition. Specialized markers of self are expressed constitutively on normal healthy cells of the host and, by engaging inhibitory receptors, prevent phagocytosis of these cells by macrophages, killing by NK cells, and perhaps phagocytosis and presentation of antigens derived from normal healthy cells by dendritic cells. Conversely, the absence of these markers on microbial cells and their down-regulation on infected, transformed, and senescent host cells render these targets susceptible to NK-mediated cytotoxicity or phagocytosis by macrophages (Fig. 2).

Although the missing-self strategy is an efficient way to distinguish normal self from nonself or from abnormal self, it is not resistant to fraud. Indeed, in multiple examples of "stolen identity," pathogens acquire by horizontal transfer the genes encoding self-markers and thus protect themselves from detection and destruction by the host.

\section{Signals for Assisted Cellular Suicide}

Induction of apoptosis in virally infected or transformed cells is an important mechanism of host defense, as it ensures the survival of the host by preventing viral spread and the development of cancer. Apoptosis of infected or transformed cells can be induced either by a cell-autonomous mechanism, or by extracellular signals derived from other cells (14, 15). For example, viral infection or excessive DNA damage trigger apoptosis in the affected cells through PKR/RNaseL- and p53-dependent pathways, respectively. Cell-autonomous cell death is presumably also part of the early host defense against cellular transformation (16). In other cases, infection, transformation, and excessive stress do not induce apoptosis directly, but instead induce the expression of molecular markers that flag the affected cells for killing by specialized executioners, most commonly, NK cells. Several of these markers have been described, including human MICA and ULBP proteins, and

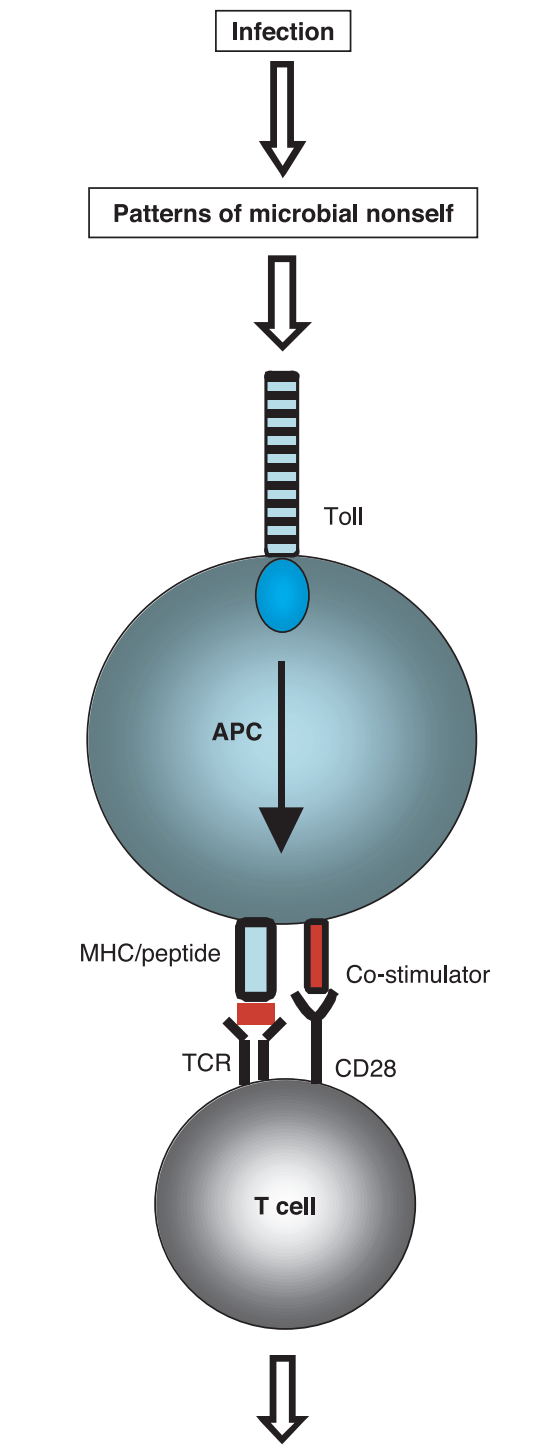

Activation of the adaptive immune response

Fig. 1. Toll-like receptors (TLRs) are triggered by markers of microbial nonself (PAMPs) to induce the expression of host defense genes, as well as costimulatory molecules on the surface of APCs. Costimulatory molecules, along with pathogen-derived peptides presented on MHC molecules, are necessary for the activation of naïve $\mathrm{T}$ cells. 


\section{REFLECTIONS ON SELF: IMMUNITY AND BEYOND}

mouse RAE-1 and H-60 proteins (17-19). Intriguingly, all these proteins are distant relatives of the MHC class-I proteins and all of them serve as signals for assisted suicide by triggering an activating receptor, NKG2D, expressed on NK cells, CD8-positive T cells and myeloid cells $(17-20)$. Unlike inhibitory receptors, the activating receptors lack ITIM motifs but generally are coupled with adapters that trigger either ZAP-70/Syk tyrosine kinases, or activation pathways that are dependent on phosphatidylinositol 3-kinase (21). These pathways are subject to negative regulation by the SHP-1/SHP-2 tyrosine phosphatases recruited by inhibitory receptors (8). Moreover, the susceptibility of the target cell to killing by NK cells depends on the balance of the ligands for activating and inhibitory receptors $(21)$. This is reminiscent of the balance between proapoptotic and antiapoptotic members of the Bcl-2 family proteins that control intrinsic apoptosis (15) and presumably reflects the fact that "fuzzy" decision-making mechanisms are more resistant to errors than discrete, all-or-none processes.

The existence of inducible markers of abnormal self that flag cells for assisted apoptosis raises an important question: because in- fection, stress, and transformation can induce cell-autonomous apoptosis, why do cells bother with expressing these ligands and requesting NK cell assistance at all? There are several possible answers to this question. First, recognition of these ligands by NK and $\mathrm{CD}^{+} \mathrm{T}$ cells does not lead just to apoptosis of the target cell; it also activates the NK and $\mathrm{CD}^{+} \mathrm{T}$ cells to produce cytokines such as interferon- $\gamma$. The second possibility is that the involvement of the extrinsic apoptotic pathway may help to counteract viral strategies of blocking the intrinsic pathway (22) and to bypass possible blocks in the cell autonomous pathway caused by mutations in tumor cells.

\section{Other Markers of Abnormal Self}

The inducible signals that recruit specialized killers are not the only markers of abnormal self. Other important and frequently used signals have to do with recognition of the two types of cell death-necrosis and apoptosis. The enormous number of apoptotic cells produced during the life of a metazoan animal indicates that the recognition and removal of apoptotic cells is a highly efficient and vitally important process. Apoptotic cells are phago-
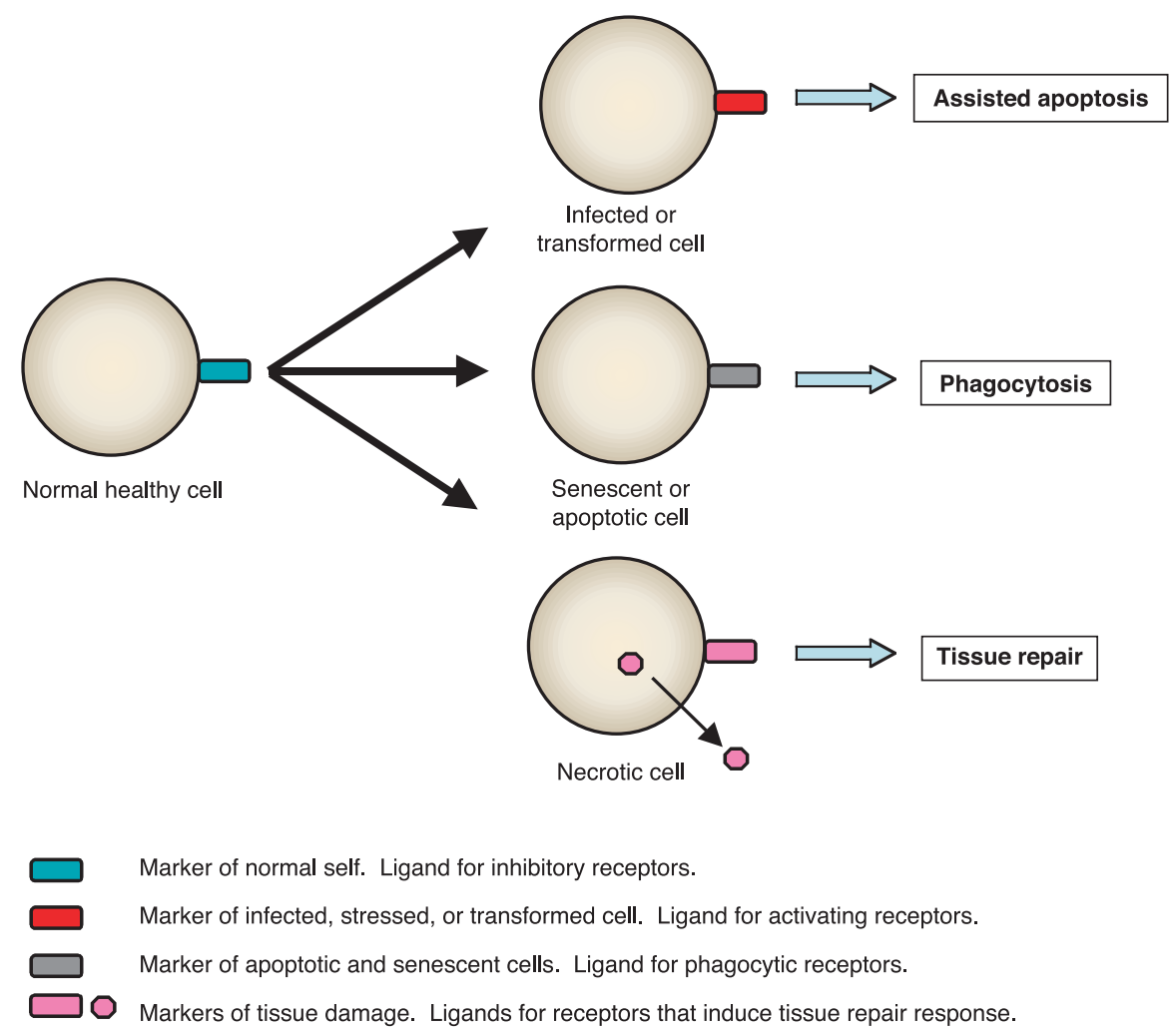

Fig. 2. Normal healthy cells constitutively express markers of self, which engage inhibitory receptors on the effector cells of the immune system. Infected, transformed, or damaged cells down-regulate the expression of these markers, and induce the expression of ligands for activating receptors on NK and other effector cells. Apoptotic or senescent cells display markers that tag them for phagocytosis by macrophages, whereas necrotic cells express signals that induce tissue repair responses. Appropriate expression of these markers enables cells of the innate immune system to discriminate between normal, healthy cells and nonself/abnormal cells, and to ignore, phagocytose, or kill them as needed.

cytosed by neighboring cells, or more commonly by macrophages, because they express a number of cell surface markers recognized by cells that phagocytose them. The most important marker appears to be phosphatidylserine (PS), which in normal live cells is found on the inner leaflet of the plasma membrane, but in apoptotic cells is localized to the outer leaflet where it can be recognized by a PS receptor (23) (Fig. 2). Recognition of PS by the PS receptor leads to phagocytosis of apoptotic cells, while sparing healthy, live cells (23).

Recognition of necrotic cells is more complicated because the markers of necrosis have yet to be characterized in detail. During necrosis, intracellular components that are normally hidden inside the cell are released and induce inflammation. There is, however, a substantial difference in opinion regarding the purpose and the outcome of necrosis-induced inflammation. One model maintains that necrosis, and tissue damage in general, provides a danger signal that functions as the major inducer of immune responses (24). The problem with this mod$\mathrm{el}$, in our opinion, is its inherent tautology. According to this hypothesis, the immune response is induced by a danger signal, but the danger signal is defined as just about anything that can induce an immune response. We believe that the major physiological role of necrosis-induced inflammation is to induce a tissue repair response, as tissue repair seems to be the most logical response to tissue damage. The induction of an immune response, on the other hand, is controlled by the innate immune system, which detects the signs of infection by decoding the patterns of self and nonself.

\section{References and Notes}

1. C. A. Janeway Jr., Cold Spring Harb. Symp. Quant. Biol. 54, 1 (1989).

2. R. Medzhitov, C. A. Janeway Jr., Cell 91, 295 (1997).

3. R. Medzhitov, Nature Rev. Immunol. 1, 135 (2001).

4. K. Karre, H. G. Ljunggren, G. Piontek, R. Kiessling, Nature 319, 675 (1986).

5. L. L. Lanier, Annu. Rev. Immunol. 16, 359 (1998).

6. E. O. Long, Annu. Rev. Immunol. 17, 875 (1999).

7. A. Diefenbach, D. H. Raulet, Immunol. Rev. 181, 170 (2001).

8. J. V. Ravetch, L. L. Lanier, Science 290, 84 (2000).

9. K. F. Austen, D. T. Fearon, Adv. Exp. Med. Biol. 120B, 3 (1979).

10. P. R. Crocker, A. Varki, Trends Immunol. 22, 337 (2001).

11. S. Kelm, R. Schauer, Int. Rev. Cytol. 175, 137 (1997).

12. G. M. Doody et al., Science 269, 242 (1995).

13. P. A. Oldenborg et al., Science 288, 2051 (2000).

14. D. R. Green, J. C. Reed, Science 281, 1309 (1998).

15. J. M. Adams, S. Cory, Science 281, 1322 (1998).

16. G. Evan, T. Littlewood, Science 281, 1317 (1998).

17. A. Cerwenka et al., Immunity 12, 721 (2000).

18. A. Diefenbach, A. M. Jamieson, S. D. Liu, N. Shastri, D. H. Raulet, Nature Immunol. 1, 119 (2000).

19. D. Cosman et al., Immunity 14, 123 (2001).

20. M. Girardi et al., Science 294, 605 (2001).

21. L. L. Lanier, Nature Immunol. 2, 23 (2001).

22. D. L. Vaux, G. Haecker, A. Strasser, Cell 76, 777 (1994).

23. J. Savill, V. Fadok, Nature 407, 784 (2000).

24. P. Matzinger, Science 296, 301 (2002).

25. Research in our laboratories is supported by the Howard Hughes Medical Institute and by grants from NIH. 